\title{
Recurrent anteroinferior glenohumeral instability resulting from a proximal humerus osteochondroma
}

\author{
Marlis T. Sabo*, Stephen J. French \\ Section of Orthopaedic Surgery, Department of Surgery, University of Calgary, Calgary, Canada
}

Received: December 10, 2016

DOI: $10.5430 /$ css.v3n 1 p 18
Accepted: January 8, 2017

Online Published: January 12, 2017

URL: http://dx.doi.org/10.5430/css.v3n1p18

\begin{abstract}
Shoulder instability is a common clinical event, with most causes due to capsulolabral pathology within the shoulder joint. We present a case of a young male with chronic recurrent anteroinferior instability due to impingement of a proximal humerus osteochondroma on the glenoid during shoulder range of motion. Excision of the osteochondroma and repair of the anteroinferior capsule to the humeral neck successfully treated the instability and enabled a rapid improvement in patient function.
\end{abstract}

Key Words: Shoulder instability, Osteochondroma, Shoulder reconstruction, Benign bone neoplasm

\section{INTRODUCTION}

Although anteroinferior shoulder instability is a common problem, chronic anteroinferior instability due to impingement of a proximal humerus osteochondroma is not. We present a case of such instability in a healthy young male patient.

\section{Case presentation}

A healthy 27-year-old male presented to clinic with a long history of shoulder dysfunction related to recurrent anteroinferior instability. His index dislocation was as an adolescent. He went on to have multiple episodes of instability, treated non-operatively with physiotherapy. Pain subsequently developed. Of note, he was found to have an osteochondroma of his proximal medial humerus (see Figure 1) during his initial treatment phase as an adolescent. This was felt to be benign after extensive investigation.

Initial clinical evaluation demonstrated no scars, deformity, or atrophy about his shoulder, with normal rotation at waist level. Substantial scapular dyskinesis was noted and he was not able to actively elevate beyond 100 degrees. Passive assistance could not bring the arm to full forward elevation. Upon closer examination, it was proposed that the osteochondroma was engaging the glenoid during range of motion. The symptomatic anteroinferior instability could be reproduced with a forceful internal rotation and adduction maneuver. Standard apprehension/relocation testing did not elicit symptoms of instability, and he had no features of increased systemic laxity.

An Magnetic Resonance Imaging (MRI) demonstrated minimal labral pathology (see Figure 2A). The osteochondroma was found to be $6.0 \mathrm{~cm} \times 2.3 \mathrm{~cm} \times 2.4 \mathrm{~cm}$ with a $2 \mathrm{~mm}$ cartilage cap and no suspicious findings (see Figure 2B). No Hill-Sachs lesion was noted.

In discussion with the patient, it was decided to perform an arthroscopic assessment of the intra-articular space followed by an open resection of the osteochondroma. At time of surgery, the entire anteroinferior labrum was normal in appearance. A dynamic maneuver bringing the arm into an abducted, externally rotated position brought the tip of the

\footnotetext{
*Correspondence: Marlis T. Sabo; Email: marlis.sabo@gmail.com; Address: Section of Orthopaedic Surgery, Department of Surgery, University of Calgary, Calgary, Canada.
} 
osteochondroma into view as it impinged on the axillary pouch (see Figure 3A). No humeral avulsion of the glenohumeral ligaments was noted. A deltopectoral approach was then employed to access the proximal humerus. The subscapularis was tenotomized and peeled off the capsule as a separate layer. The capsule was then sharply dissected off the humeral neck around to the 8 o'clock position. Sharp and blunt dissection was used to expose the osteochondroma (see Figure 3B) and retractors protected the other vital structures. The osteochondroma was excised. Repair of the capsule was performed using titanium anchors, introducing a small capsular shift, while the subscapularis tenotomy was repaired with non-absorbable suture. Rehabilitation proceeded as routine for an open shoulder procedure: range of motion exercises for 12 weeks, followed by strengthening and proprioception after 12 weeks. Pathology was consistent with a completely benign lesion.

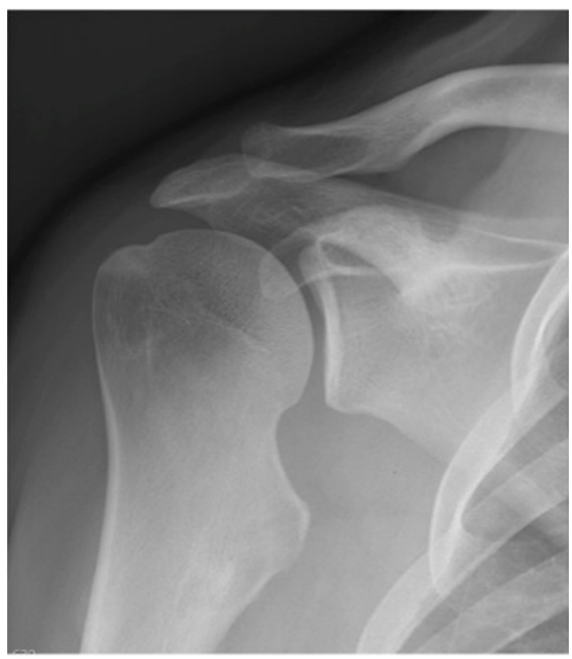

Figure 1. Anteroposterior (AP) Radiograph of the right proximal humerus

Note the large sessile osteochondroma present on the proximal medial aspect of the humerus; No other skeletal abnormality is noted.

At six months post-operatively, the patient had full range of motion in forward elevation, internal and external rotation (see Figure 4A-C) with good scapular control. He had no pain and no symptoms of instability. He had returned to his physically active work and had no further concerns.

\section{Discussion}

Osteochondromas are benign chondroid lesions that arise during skeletal growth and usually become dormant when growth is complete. ${ }^{[1]}$ Although generally asymptomatic, there are locations in which mass effects and irritative effects can cause problems. ${ }^{[1]}$ Examples include painful snapping scapula due to scapular osteochondromas ${ }^{[2]}$ and popliteal Published by Sciedu Press pseudoaneurysms from tumors around the knee. ${ }^{[3]}$ In the forearm, dynamic impingement and growth disturbance between the forearm bones may also lead to deformity, altered rotation. ${ }^{[4]}$ Osteochondromas are well-known in the proximal humerus with a variety of effects. Pain is a common symptoms, and one avenue of pain generation is formation of an abnormal bursa over the stalk and cap resulting in pain and even mass formation as the bursa fills with fluid. ${ }^{[5,6]}$ In another example, an osteochondroma arising from the bicipital groove created biceps tendinitis. ${ }^{[7]}$ Neurovascular syndromes may be created because the osteochondroma is a space-occupying lesion. Quadrilateral space syndrome has been seen with proximal humerus osteochondromas. ${ }^{[8]}$ Loss of glenohumeral motion has also been observed when unusual locations of osteochondromas within the shoulder joint capsule have created mechanical blocks to motion. ${ }^{[9,10]}$ Joint dislocation has been described not in the shoulder joint, but in the elbow joint complex, usually as the result of a deformity or mass effect arising either on the radius or the ulna. ${ }^{[11,12]}$ This does not represent a dynamic process, but rather a static progressive joint dislocation created by the mass, which was successfully reversed by excision and joint reduction in these two young pediatric patients.
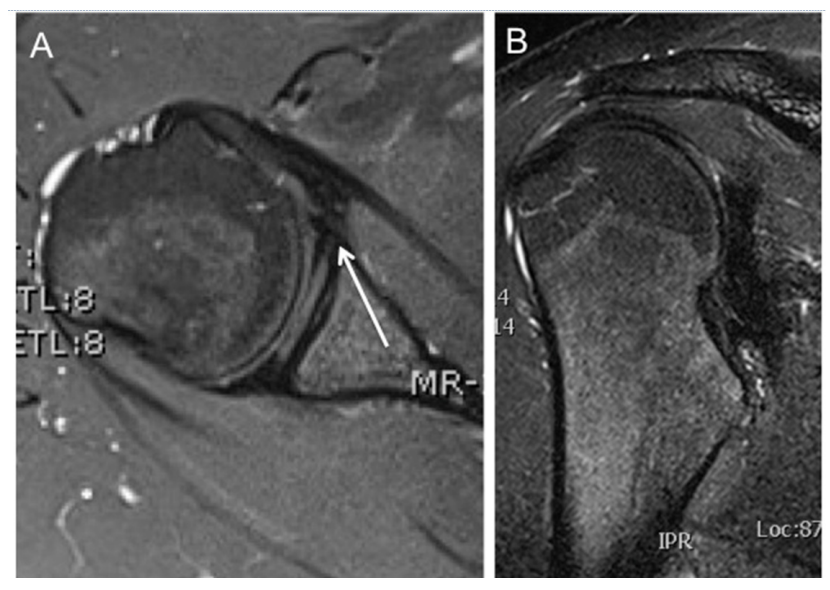

Figure 2. Representative T2 sections of the MRI scan A. Axial image of the anteroinferior labrum. No significant blunting or tearing of the labrum is evident (white arrow); B. Coronal image of the shoulder joint and proximal humerus demonstrating the osteochondroma, The cartilage cap is thin.

Excision of proximal humerus osteochondromas is most often for pain or stiffness and is generally successful. Bae et al. ${ }^{[13]}$ described a retrospective case series of 31 pediatric patients presenting with proximal humerus osteochondromas. In their series of skeletally immature patients, only 2 recurrences were noted, 1 being in a patient with multiple hereditary exostoses. In some of the patients, the lesion size and location precluded a full excision. 

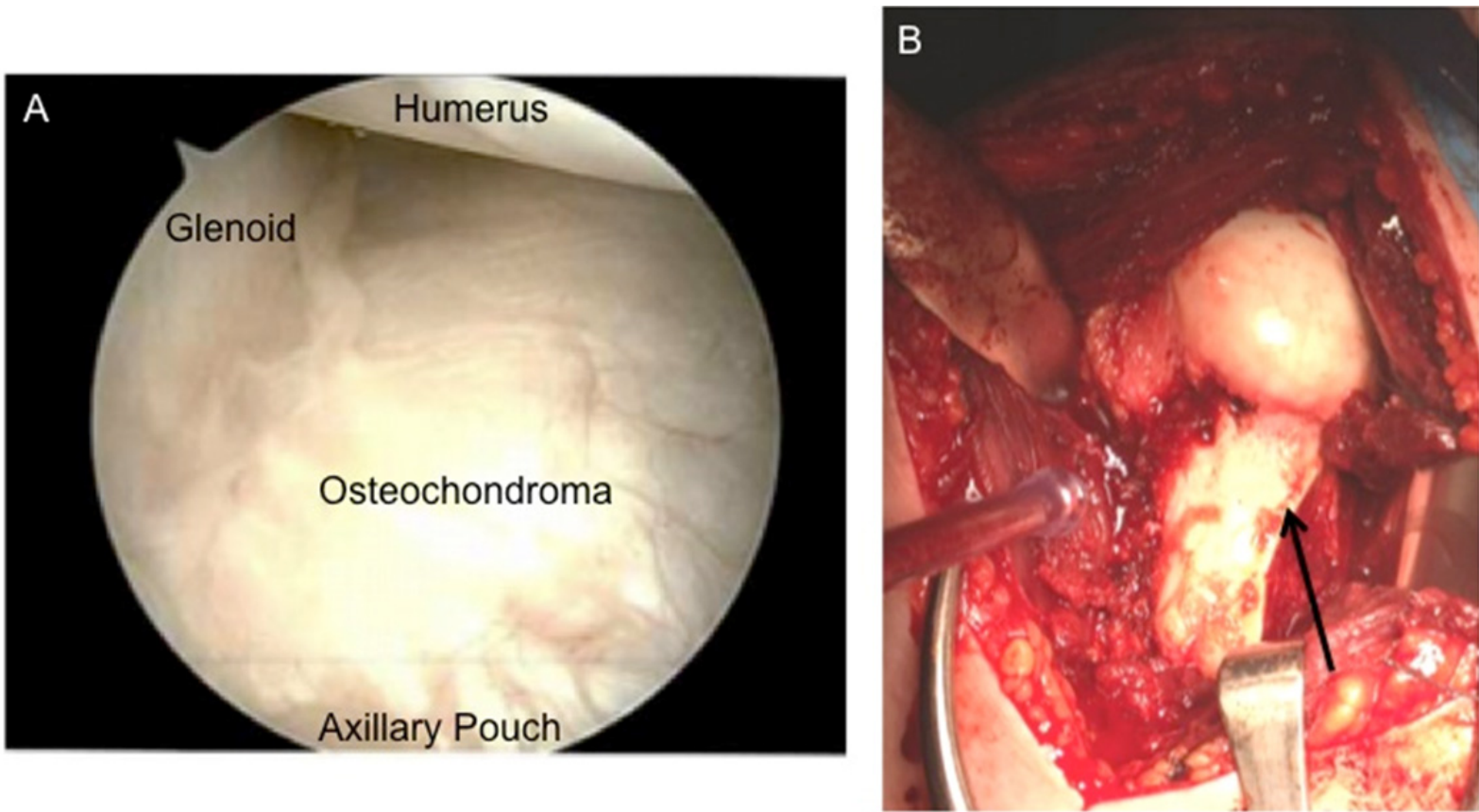

Figure 3. Intraoperative images of the right shoulder

A. Arthroscopic image with the camera in the posterior viewing portal and in the axillary pouch. The pouch is indented by the osteochondroma as the arm is brought into an abducted and externally rotated position; B. Image of the exposed osteochondroma (black arrow).
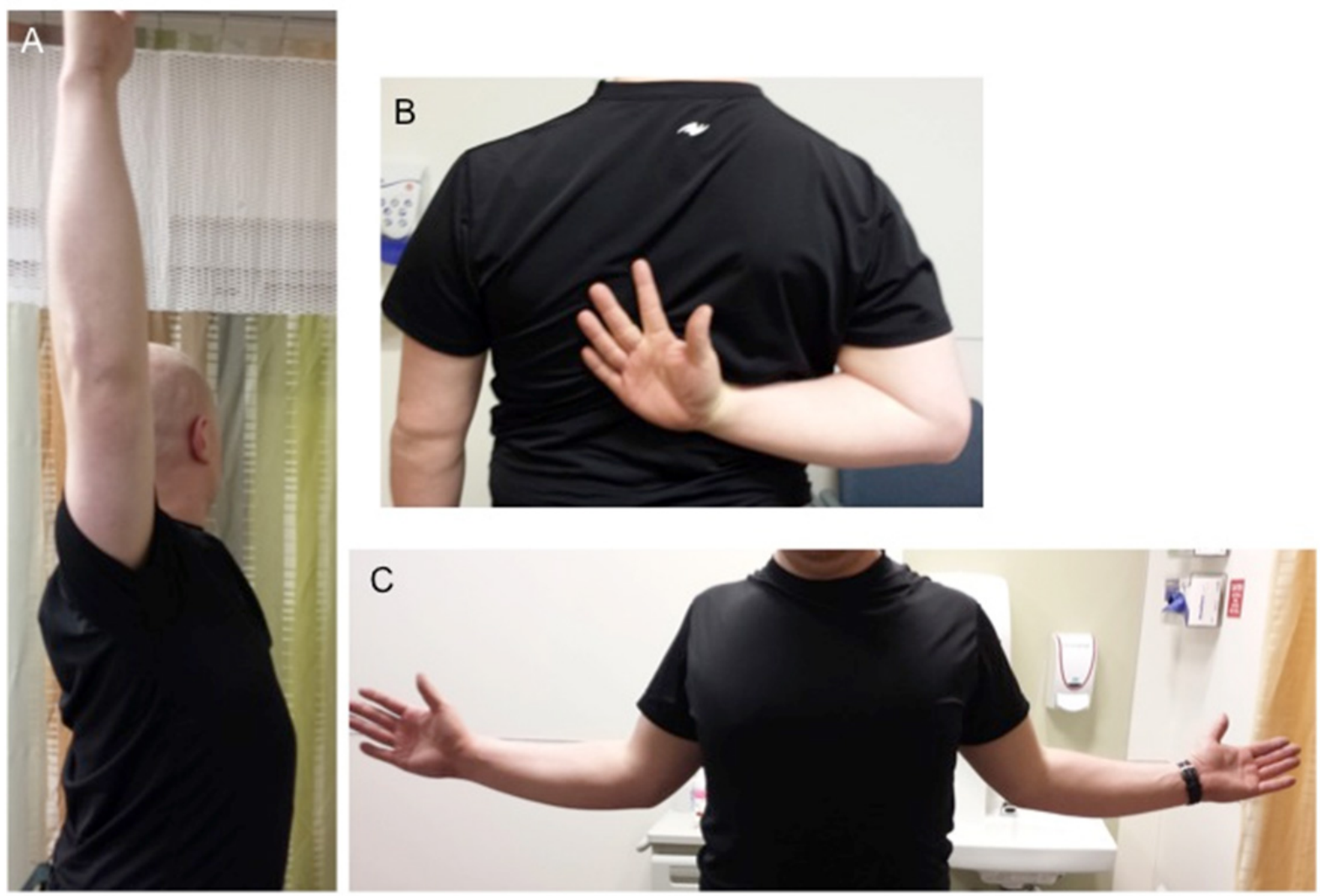

Figure 4. Post-operative range of motion

The patient had full and symmetric forward elevation $(A)$, internal rotation $(B)$ and external rotation $(C)$ in the operative arm. 
The situation faced by our patient was somewhat different from the above, being a dynamic shoulder instability due to impingement of the osteochondroma combined with muscle patterning. This patient also displayed clinical features that were atypical for classic anteroinferior shoulder instability: the typical examination findings of anterior apprehension corrected by the relocation maneuver were absent. The classic imaging findings that accompany recurrent anteroinferior shoulder instability were also absent; the anterior labrum was in good condition. Furthermore, his capsule was not notably patulous. Treatment algorithms for shoulder instability aim to address the pathology present. For example, if a Bankart lesion (tear of anteroinferior capsulolabral complex) is present, repair of the labral structures onto the glenoid face is preferred. Similarly, if a patient presents with atraumatic instability in multiple directions combined with a patulous capsule, procedures to reduce capsular volume have been widely employed. ${ }^{[14]}$ In this patient, the major identifiable structural pathology was the osteochondroma, and so efforts were directed to removing this structural lesion.

This case also highlights the fact that instability is often a dynamic process, whereas imaging is static and rarely places joints and limbs in positions likely to provoke instability. This man's imaging did not bring the impinging structures into contact. When confronted with unusual clinical situations, thinking beyond typical patterns of presentation can be both important and helpful to arriving at a successful treatment plan. Addressing the osteochondroma proved essential to success for this patient.

Anteroinferior shoulder instability resulting from osteochondroma impingement is a rare event dependent on the location, size, and muscle activity in the patient. Excising the osteochondroma with appropriate soft tissue closure can effectively treat the instability.

\section{CONFLicts OF INTEREST Disclosure}

The authors declare they have no conflict of interest.

\section{REFERENCES}

[1] Qasem SA, DeYoung BR. Cartilage-forming tumors. Semin Diagn Pathol. 2014; 31: 10-20. https://doi.org/10.1053/j . semdp. 2014.01 .006

[2] van Riet RP, Van Glabbeek F. Arthroscopic resection of a symptomatic snapping subscapular osteochondroma. Acta Orthop Belg. 2007; 73: 252-4. PMid: 17515241.

[3] Wiater JM, Farley FA. Popliteal pseudoaneurysm caused by an adjacent osteochondroma: a case report and review of the literature. Am J Orthop (Belle Mead NJ). 1999; 28: 412-6. PMid: 10426440.

[4] Gottschalk HP, Kanauchi Y, Bednar MS, et al. Effect of osteochondroma location on forearm deformity in patients with multiple hereditary osteochondromatosis. J Hand Surg Am. 2012; 37: 2286-93.

[5] De Groote J, Geerts B, Mermuys K, et al. Ostochondroma of the proximal humerus with frictional bursitis and secondary synovial osteochondromatosis. JBR-BTR. 2015; 98: 45-7. PMid: 26223067.

[6] Ozdemir MZ, Karakaplan M, Kahraman AS, et al. Adventitious bursitis overlying an osteochondroma of the humerus facing the thoracic wall. Case Rep Radiol. 2013; 2013: 939372.

[7] Onga T, Yamamoto T, Akisue T, et al. Biceps tendinitis caused by an osteochondroma in the bicipital groove: a rare cause of shoulder pain in a baseball player. Clin Orthop Relat Res. 2005; 431: 241-4. https://doi.org/10.1097/01.blo.0000146542.46031.f6

[8] Cirpar M, Gudemez E, Cetik O, et al. Quadrilateral space syndrome caused by a humeral osteochondroma: a case report and

review of literature. HSS J. 2006; 2: 154-6. PMid: 18751829. https://doi .org/10.1007/s11420-006-9019-y

[9] Lee JY, Lee S, Joo KB, et al. Intraarticular osteochondroma of shoulder: a case report. Clin Imaging. 2013; 37: 379-81. PMid: 23465997. https://doi.org/10.1016/j.clinimag.2012.06.005

[10] Padua R, Castagna A, Ceccarelli E, et al. Intracapsular osteochondroma of the humeral head in an adult causing restriction of motion: a case report. J Shoulder Elbow Surg. 2009; 18: e30-1. PMid: 19131258. https://doi.org/10.1016/j.jse.2008.09.008

[11] Garcia-Mata S, Hidalgo-Ovejero AM. Developmental anterior dislocation of the radial head resulting from a congenital solitary osteochondroma of the proximal ulna in an infant. Bull NYU Hosp Jt Dis. 2010; 68: 38-42. PMid: 20345362.

[12] Niu XF, Yi JH, Hu J, et al. Chronic radial head dislocation caused by a rare solitary osteochondroma of the proximal radius in a child: a case report and review of the literature. BMC Res Notes. 2015; 6C: 126-8. https://doi .org/10.1186/s13104-015-1095-y

[13] Bae DS, Kim JM, Reidler JS, et al. Surgical treatment of osteochondroma of the proximal humerus: radiographic and early clinical results. J Pediatr Orthop. 2014; 34: 529-33. PMid: 24248587. https://doi.org/10.1097/BPO.0000000000000127

[14] Levy DM, Cole BJ, Bach BR Jr. History of surgical intervention of anterior shoulder instability. J Shoulder Elbow Surg. 2016; 25: e139-150. PMid: 27066962. https://doi.org/10.1016/j.jse. 2016.01 .019 